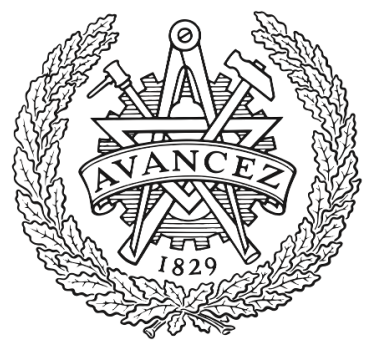

CHALMERS

UNIVERSITY OF TECHNOLOGY

\title{
Angular Dependence of InP High Electron Mobility Transistors for Cryogenic Low Noise Amplifiers under a magnetic field
}

Downloaded from: https://research.chalmers.se, 2023-04-26 12:41 UTC

Citation for the original published paper (version of record):

Harrysson Rodrigues, I., Niepce, D., Moschetti, G. et al (2019). Angular Dependence of InP High Electron Mobility Transistors for Cryogenic Low Noise Amplifiers under a magnetic field. IIS UTokyo SYMPOSIUM No.100

N.B. When citing this work, cite the original published paper. 


\title{
Angular Dependence of InP High Electron Mobility Transistors for Cryogenic Low Noise Amplifiers under a magnetic field
}

\author{
I. H. Rodrigues*1, D. Niepce ${ }^{1}$, G. Moschetti ${ }^{2}$, A. Pourkabirian², J. Schleeh², T. Bauch ${ }^{1}$, J.Grahn ${ }^{1}$ \\ ${ }^{1}$ Department of Microtechnology and Nanoscience - MC2, Chalmers University of Technology, SE-41296 \\ Gothenburg, Sweden \\ ${ }^{2}$ Low Noise Factory AB, Nellickevägen 22, Gothenburg, SE-41663, Sweden \\ * isabelr@chalmers.se
}

This work addresses the angular dependence of DC properties in 100nm InP HEMT devices under the influence of applied static magnetic field at $2 \mathrm{~K}$. When kept at an angle $90^{\circ}$ towards a magnetic field of $14 \mathrm{~T}$, the maximum output drain current $I_{d s}$ was reduced more than $99 \%$. A rotation sweep of the transistor revealed a strong angular and Bfield dependence on $I_{d s}$. This was correlated with a reduction in dc transconductance and increase in on-resistance of the transistor. The RF properties of the transistor were tested by measuring an 0.3-14 GHz InP HEMT MMIC lownoise amplifier (LNA) at $2 \mathrm{~K}$ kept at an angle $90^{\circ}$ towards a magnetic field up to $10 \mathrm{~T}$. The gain and noise temperature were strongly decreased and increased, respectively, already below $1 \mathrm{~T}$. The results show that precise alignment of the cryogenic InP HEMT LNA is crucial in a magnetic field. Even a slight mis-orientation of a few degrees leads to a strong degradation of the gain and noise temperature.

Keywords - InP HEMT; low noise amplifier; cryogenic; angular dependence; magnetic field;

The InP HEMT was recently reported to be strongly affected when aligned perpendicular (or $\left.90^{\circ}\right)$-with respect to a magnetic field up to $2 \mathrm{~T}$ [1]. This has implications on the alignment of cryogenic InP HEMT low-noise amplifiers (LNAs) under strong magnetic fields. Such ultra-sensitive microwave receivers are used in detector experiments of dark matter [2] and mass spectrometry [3]. A potential application is MRI where cryogenic LNAs can provide increased sensitivity [4]. Previous reports on the cryogenic LNA ha only reported on 0 or $90^{\circ}$ orientation versus magnetic field [5]. We have here for the first time studied the angular dependence from 0 to $90^{\circ}$ of the cryogenic InP HEMT under applied magnetic fields.

In Fig. 1. a schematic illustration of the experiment is shown. All measurements were carried out at an ambient temperature of $2 \mathrm{~K}$. An external magnetic field was applied with an angle $\theta$ relative to the two-dimensional electron gas (2DEG) of the device which was a $130 \mathrm{~nm}$ In HEMT [6]. Fig. 2. (a)-(c) shows the output drain current $\mathrm{I}_{\mathrm{ds}}$ at different gate voltages $\mathrm{V}_{\mathrm{gs}}$ for an applied field of 0,7 or $14 \mathrm{~T}$. It is clear that the output drain current is drastically reduced with increased field, also illustrated in Fig. 2 (d) using normalized $\mathrm{I}_{\mathrm{ds}}$ as a function of B. Furthermore, the dc transconductance as a function of magnetic field for different $\theta$ showed the same trend; see Fig. 3 (a). The on-resistance $\mathrm{R}_{\text {on }}$ versus magnetic field strongly increased, also dependent on $\theta$; see Fig. 3 (b). Devices with different gate lengths $(60,100$ and $250 \mathrm{~nm})$ and different gate widths $(2 \times 10,2 \times 50$ and $2 \times 100 \mu \mathrm{m})$ when aligned perpendicular to the applied magnetic fields showed no difference with respect to $\mathrm{I}_{\mathrm{ds}}(\mathrm{B})$ dependence; see Fig 4. Rotating the sample from $\theta=0$ to $180^{\circ}$ revealed a field- and angular dependence; see Fig. 5. The dependence can be expressed using $V_{d s}$, magnetic field $B, \theta$, a channel resistance $R_{0}$ and a fitting parameter A: $\mathrm{I}_{\mathrm{ds}}(\mathrm{B}, \theta)=\mathrm{V}_{\mathrm{ds}} / \mathrm{R}_{\mathrm{xx}}=\mathrm{V}_{\mathrm{ds}} /\left(\mathrm{R}_{0}+\mathrm{A}(\sin (\theta) \mathrm{B})^{2}\right)$. In Fig. 5 the fitting is plotted for $\mathrm{V}_{\mathrm{gs}}=\mathrm{V}_{\mathrm{ds}}=0.4 \mathrm{~V}$.

The RF properties of the InP HEMT were examined by measuring an 0.3-14 GHz InP HEMT MMIC LNA kept at $2 \mathrm{~K}$ in a perpendicular $\left(\theta=90^{\circ}\right)$ magnetic field up to $10 \mathrm{~T}$. The InP HEMT in the MMIC LNA was fabricated in the same technology as the device measured in the DC experiments described above. Fig. 6. shows that the gain decreased and the noise strongly increased already up to $1 \mathrm{~T}$ of.

This study reveals the importance of precise alignment of the InP HEMT in a magnetic field, to avoid a strong degradation of noise performance of the cryogenic InP HEMTs LNA.

References: [1] I. H. Rodrigues et al., "Magnetic Influence on Cryogenic InP HEMT DC Characteristics," Compond Seiconductor Week, Boston (2018)

[2] C. Hagmann et al., "Results from a search for cosmic axions", Phys. Rev. D 42, 1297 (1990).

[3] R. Mathur, et al., "A Low-Noise Broadband Cryogenic Preamplifier Operated in a High-Field Superconducting Magnet", IEEE Trans. Appl. Superconductivity 18, 1781 (2008).

[4] D. H. Johansen et al., "Cryogenic Preamplifiers for Magnetic Resonance Imaging," IEEE Trans Biomed. Circuits Systems $12,202(2018)$

[5] E. Daw and R. F. Bradley, "Effect of high magnetic fields on the noise temperature of a heterostructure field-effect transistor low-noise amplifier", J. Appl. Phys. 82, 1925 (1997).

[6] J. Scleeh et al., Characterization and Modeling of Cryogenic Ultralow-Noise InP HEMTs", IEEE Trans. Electron. Dev. 60, 206 (2013). 


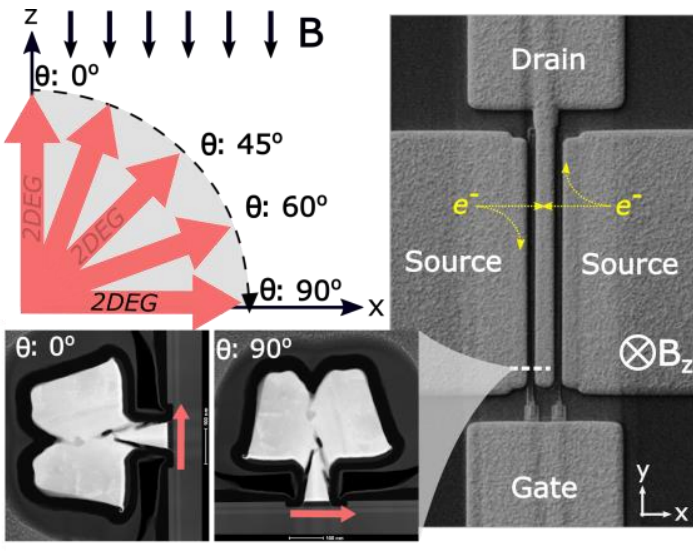

Fig. 1. Schematic image of experiment (top left), where an external magnetic field is applied and the 2DEG in the device, is aligned and rotated with an angle $\theta$ relative to the direction of the field. SEM image shown of cross section of gate (bottom left) and top view of transistor (right).
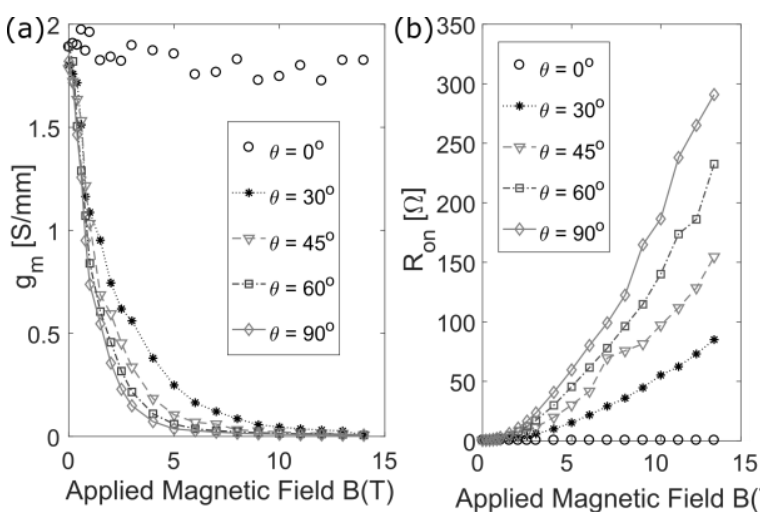

Applied Magnetic Field B(T)

Fig. 3. (a) Transconductance as a function of applied static magnetic field ranging from 0 to 14 , applied at various $\theta: 0^{\circ}, 30^{\circ}$, $45^{\circ}, 60^{\circ}, 90^{\circ}$. (b) $R_{\text {on }}$ versus magnetic field for the same configurations. $\mathrm{T}=2 \mathrm{~K} . \mathrm{L}_{\mathrm{g}}=100 \mathrm{~nm}$ and $\mathrm{W}_{\mathrm{g}}=2 \times 50 \mu \mathrm{m}$.

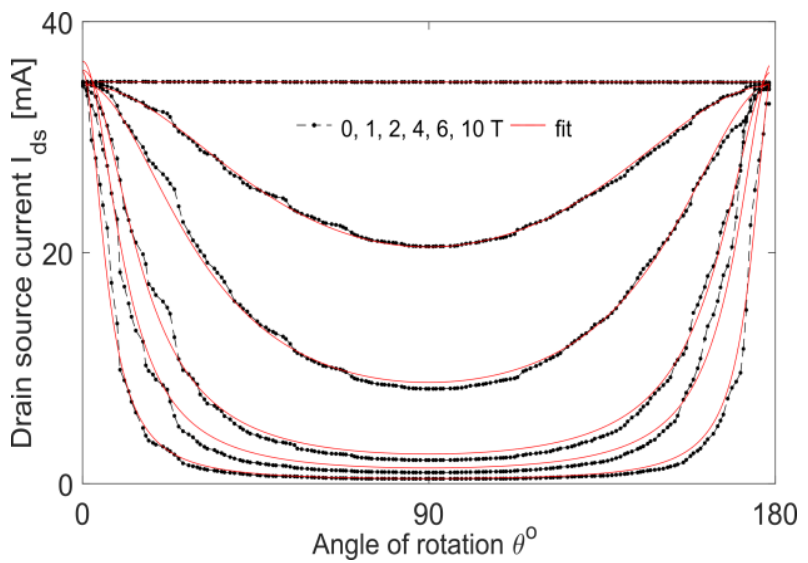

Fig. 5. Fit of rotation sweep. Drain current versus angle of rotation for various applied static magnetic field ranging from 0 to $10 \mathrm{~T}$ and biased with $\mathrm{V}_{\mathrm{gs}}$ and $\mathrm{V}_{\mathrm{ds}}$ of $0.4 \mathrm{~V}$ respectively. $\mathrm{T}=2 \mathrm{~K}$. Solid line shows a fit of rotation sweep using Eq. (1)
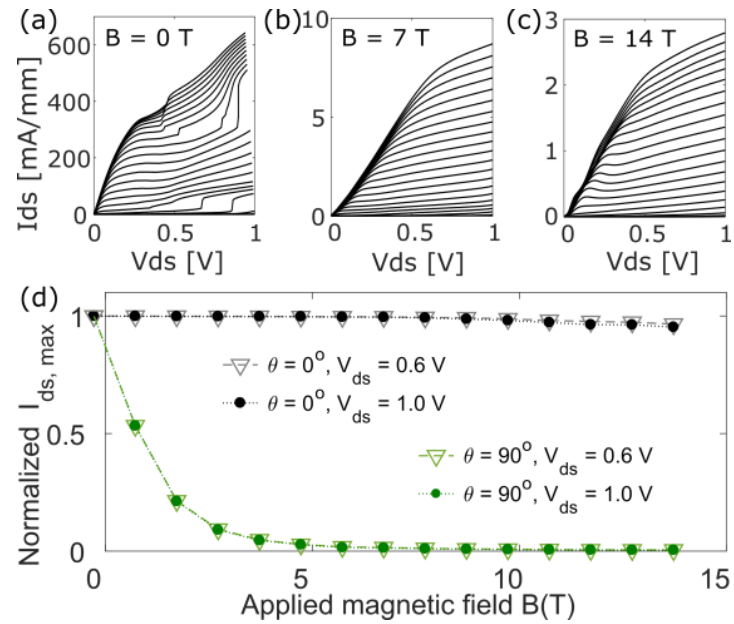

Fig. 2. IV-characteristics with (a) no applied field, (b) an applied field of $7 \mathrm{~T}$ and (c) an applied field of $14 \mathrm{~T}$. (d) The normalized output drain current versus field. $\mathrm{T}=2 \mathrm{~K}$. $\mathrm{V}_{\mathrm{gs}}:-0.4$ to $0.4 \mathrm{~V}, \mathrm{~L}_{\mathrm{g}}=$ $100 \mathrm{~nm}$ and $\mathrm{W}_{\mathrm{g}}=2 \times 50 \mu \mathrm{m}$
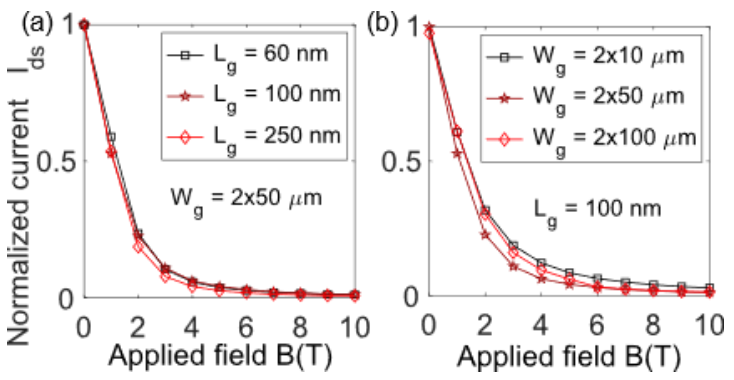

Fig. 4. Normalized $I_{d s}$ for various devices, with (a) different gate length and (b) different gate width, when aligned perpendicular to the applied magnetic fields ranging from 0 to $10 \mathrm{~T}$. A fix $\mathrm{V}_{\mathrm{gs}}$ and $\mathrm{V}_{\mathrm{ds}}$ of $0.4 \mathrm{~V}$ was applied, in an ambient temperature of $2 \mathrm{~K}$.

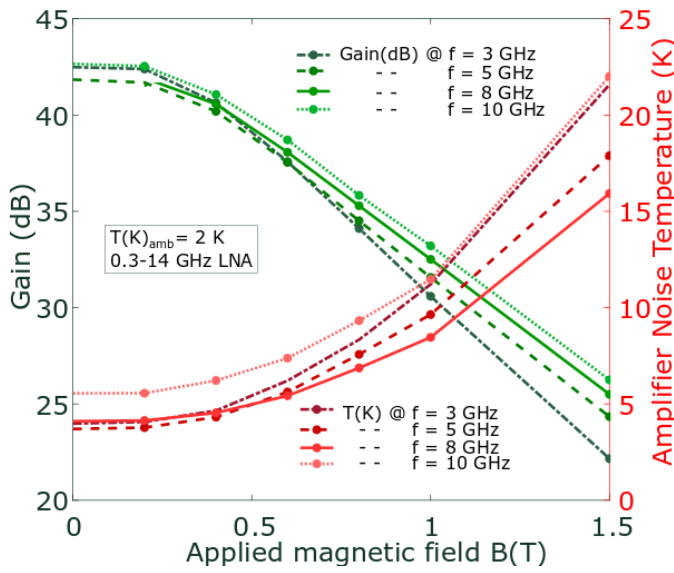

Fig. 6. Experimental set-up for a) gain measurements and b) noise measurements. Gain (dB) (left y-axis) and Noise temperature $\mathrm{T}(\mathrm{K})$ (right y-axis) of the cryogenic InP LNA measured at $2 \mathrm{~K}$, when increasing applied magnetic field from 0 to $1.5 \mathrm{~T}$. 